\title{
Consensus Dynamics on Random Rectangular Graphs
}

\author{
Ernesto Estrada ${ }^{1,2}$ and Matthew Sheerin ${ }^{1}$
}

${ }^{1}$ Department of Mathematics 83 Statistics, University of Strathclyde, 26 Richmond Street, Glasgow G1 1XQ, U. K.; ${ }^{2}$ Centre for Mathematical Research (CIMAT), Guanajuato,

Mexico.

Corresponding author: Ernesto Estrada

Telf. $+44(0) 1415483657$

Fax. +44 (0)141 5483345

E-mail: ernesto.estrada@strath.ac.uk 


\begin{abstract}
A random rectangular graph ( $\mathrm{RRG}$ ) is a generalization of the random geometric graph (RGG) in which the nodes are embedded into a rectangle with side lengths $a$ and $b=1 / a$, instead of on a unit square $[0,1]^{2}$. Two nodes are then connected if and only if they are separated at a Euclidean distance smaller than or equal to a certain threshold radius $r$. When $a=1$ the RRG is identical to the RGG. Here we apply the consensus dynamics model to the RRG. Our main result is a lower bound for the time of consensus, i.e., the time at which the network reaches a global consensus state. To prove this result we need first to find an upper bound for the algebraic connectivity of the RRG, i.e., the second smallest eigenvalue of the combinatorial Laplacian of the graph. This bound is based on a tight lower bound found for the graph diameter. Our results prove that as the rectangle in which the nodes are embedded becomes more elongated, the RRG becomes a 'large-world', i.e., the diameter grows to infinity, and a poorly-connected graph, i.e., the algebraic connectivity decays to zero. The main consequence of these findings is the proof that the time of consensus in RRGs grows to infinity as the rectangle become more elongated. In closing, consensus dynamics in RRGs strongly depend on the geometric characteristics of the embedding space, and reaching the consensus state becomes more difficult as the rectangle is more elongated.
\end{abstract}

Keywords

- Consensus dynamics

- Random geometric graphs

- Graph diameter

- Algebraic connectivity

- Graph Laplacian 


\section{Introduction}

Many real-world networked systems are embedded into geometrical spaces. These spatial networks, as they are known, may represent many different kinds of scenarios [1]. For instance, in urban street networks the nodes describe the intersection of streets, which are represented by the edges of the graph. These streets and their intersections are embedded in the two-dimensional space representing the surface occupied by the corresponding city. Similar situations occur with infrastructural and transportation systems ranging from water supply networks and railroads to the internet and wireless sensor networks (WSNs). In WSNs [2], the nodes represent the sensors which are deployed on a given geographical region and their communication defines the connectivity of the nodes. This is analogous to many other communication systems ranging from mobile phones to radio signals. On a different scale we can mention the vascular and cellular networks of nodes embedded into cells and biological tissues [3]; protein residue networks [3]; the networks of channels in fractured rocks [4]; the networks representing the corridors and galleries in animal nests [5, 6]; and landscape networks [7], among others. For modeling these spatial networks it is necessary to have a theoretical model that captures both the topological features typical of complex networks and the spatial embedding of these specific kinds of systems. The most commonly used model for spatial networks is the so-called random geometric graph (RGG) [8, 9, 10, 11]. In RGGs each node is randomly assigned geometric coordinates and then two nodes are connected if the (Euclidean) distance between them is smaller than or equal to a certain threshold $r$.

The RGG model has been widely used in the study of wireless sensor networks (WSNs) and peer-to-peer networks [12, 13, 14], where the problem of consensus has received great attention due to the fact that it allows the achieving of tasks with a minimum overhead of communication [15, 16, 17, 18, 19]. In the consensus protocols, as they are known in technological applications, the problem consists of making the scalar states of a set of agents converge to the same value under local communication constraints [20, 21]. Thus, since the communication requires only local information there is no congestion due to network traffic. RGGs are also used to model populations which are geographically constrained in a certain region, like a city. This scenario is important, for instance, for the analysis of epidemic spreading in such populations $[22,17,23,24]$. In this sense Riley et al. [25] have remarked that RGGs "provide a nice way of escaping the lack of local correlation and clustering that are implicit properties of the configuration graphs often used to explore epidemic dynamics". In a similar fashion, RGGs can be used to model structured populations in which opinions, instead of viruses, are propagated. In this case the RGGs also captures very well the geographic constraints of the population and, in comparison with other models [26], they "are more realistic for a number of reasons: (i) $R G G$ is isotropic (on average) while regular lattice is not; (ii) the average degree for an $R G G$ can be set to an arbitrary positive number, instead of a small fixed number for the lattice; (iii) RGGs closely capture the topology of random networks of 
short-range-connected spatially-embedded artificial agents".

In the formulation of the RGG model it is assumed that the nodes are uniformly and independently distributed on a unit square (or a higher dimensional hypercube in the general case) $[8,9]$. This unit square represents the area on which the agents are interacting to reach a consensus state, and it could be a workplace, a city, or a forest, just to mention some examples. Such a square-like area is typical of many real-world scenarios. For instance, the city of San Francisco (USA) is known as the "seven-by-seven-mile square", due to the fact that the mainland part of the city is a square of nearly $11 \mathrm{~km}$ by $11 \mathrm{~km}$. However, if we consider other cities, like Manhattan, the picture looks very different. Manhattan is 13.4 miles $(21.6 \mathrm{~km})$ long and 2.3 miles $(3.7 \mathrm{~km})$ wide, which resembles a rectangular shape instead of a square one. Based on this necessity of considering the influence of the rectangular shape on the topological and dynamical properties of the random networks deployed on these areas we have recently introduced the random rectangular graph (RRG) model [27]. In this case, the nodes are uniformly and independently distributed on a unit rectangle of given side lengths. When both sides are of the same length we recover the RGG in such a way that the RRG model generalizes the RGG one.

Here, we are interested in investigating analytically and computationally how the elongation of the rectangle in the RRG affects the consensus dynamics taking place on the nodes and edges of the networks constructed on them. We start by introducing the concept of the random rectangular graph (RRG), and continue with the description of the consensus model to be considered. Then, we state the main result of this work which proves that for a RRG with a fixed number of nodes and a given connection radius, the time for reaching consensus grows to infinity when the rectangle is very elongated. We finally support our analytic results with computational simulations for RRGs.

\section{Preliminaries}

Here we present some definitions, notations, and properties which will be used in this work (see [3]). For the basic definitions about networks the reader is directed to the literature (see for instance [3]). The notation used here is standard. For instance, $k_{i}$ designate the degree of the node $i$. The matrix $K=$ $\operatorname{diag}\left(k_{i}\right)$ designate the degree matrix of the graph and the matrix $\mathcal{L}=K-A$ is the graph Laplacian, where $A$ stands for the adjacency matrix of the graph. It has entries

$$
\mathcal{L}_{u v}=\left\{\begin{array}{ll}
k_{i} & \text { if } u=v \\
-1 & \text { if }(u, v) \in E \\
0 & \text { otherwise }
\end{array} \quad \forall u, v \in V .\right.
$$

The eigenvalues of the Laplacian matrix are denoted here by: $0=\mu_{1} \leq \mu_{2} \leq$ $\cdots \leq \mu_{n}$. If the network is connected the multiplicity of the zero eigenvalue is equal to one, i.e., $0=\mu_{1}<\mu_{2} \leq \cdots \leq \mu_{n}$ and the smallest nontrivial eigenvalue $\mu_{2}$ is known as the algebraic connectivity of graph [28, 29]. Let $U$ be 
the matrix of orthonormalized eigenvectors $\vec{\psi}_{j}$ of $\mathcal{L}$, i.e., $U=\left[\begin{array}{lll}\vec{\psi}_{1} & \cdots & \vec{\psi}_{n}\end{array}\right]$. The eigenvector $\vec{\psi}_{2}$ associated with the algebraic connectivity is known as the Fiedler vector [28]. Let $\Lambda$ be the diagonal matrix of eigenvalues of the Laplacian matrix. Then, $\mathcal{L}=U \Lambda U^{T}$.

\subsection{Random Rectangular Graphs}

The RGG is defined by distributing uniformly and independently $n$ points in the unit $d$-dimensional cube $[0,1]^{d}[8]$. Then, two points are connected by an edge if their Euclidean distance is at most $r$, which is a given fixed number known as the connection radius. That is, we create a disk of radius $r$ centred at each node, and every node inside that disk is connected to the central node. This disk plays the role of the area of influence of a given node, such as the area of coverage of a mobile or wireless sensor.

In [27] we have considered a unit hyperrectangle as the Cartesian product $\left[a_{1}, b_{1}\right] \times\left[a_{2}, b_{2}\right] \times \cdots \times\left[a_{d}, b_{d}\right]$ where $a_{i}, b_{i} \in \mathbb{R}, a_{i} \leq b_{i}$, and $1 \leq i \leq d$ instead of the unit square of the RGG. Hereafter we will restrict ourselves to the 2dimensional case, which corresponds to a rectangle of unit area. Now, the RRG has been defined by distributing uniformly and independently $n$ points in the unit rectangle $[a, b]$ and then connecting two points by an edge if their Euclidean distance is at most $r$. The rest of the construction process remains the same as for the RGG. This implies that $R R G \rightarrow R G G$ as $(a / b) \rightarrow 1$ and consequently the RRG is a generalization of the RGG.

In Fig. 1 we illustrate two RRGs with different values of the rectangle side length $a$ and the same number of nodes and edges. In the first case when $a=1$ the graph corresponds to the classical random geometric graph in which the nodes are embedded into a unit square. The second case corresponds to $a=2$ and it represents a slightly elongated rectangle. 


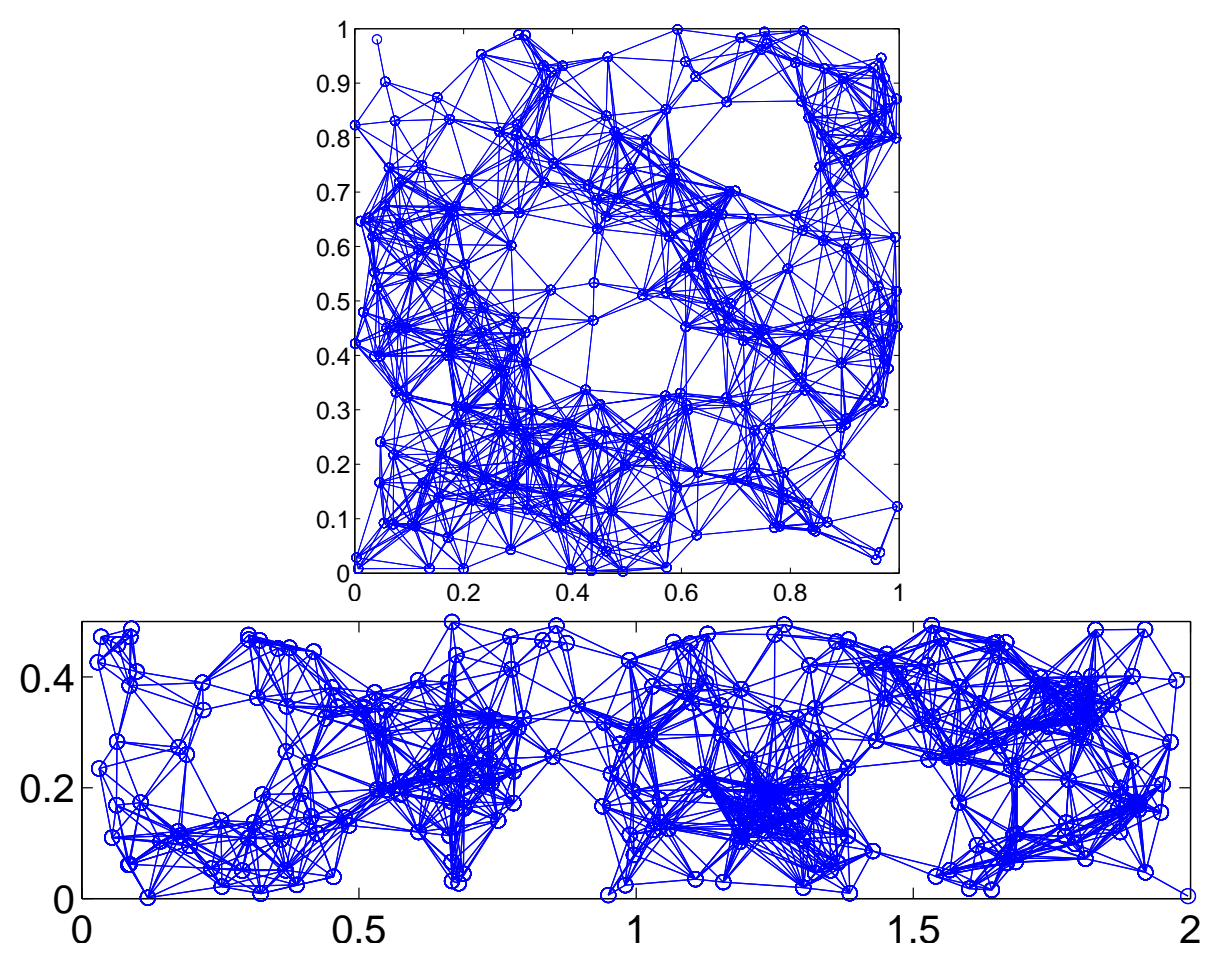

Figure 1: Illustration of a RRG created with 250 nodes embedded into a unit square, $a=1$, (top) and a unit rectangle with $a=2$ (bottom). In both cases the nodes are connected if they are at a Euclidean distance smaller than or equal to $r=0.15$.

A few important structural parameters of RRGs have been determined analytically in a previous work by the current authors (see [27]). They include the average degree, the probability that the graphs are connected, their degree distributions, average path length and clustering coefficient.

\subsection{Consensus dynamics on graphs}

Let us consider that the state of the nodes of the graph at time $t$ are stored in the vector $\vec{u}(t)$. Then, the variation of the state of the node $i$ with time is controlled by the equation $[21,20]$ :

$$
\overrightarrow{u_{i}}(t)=\sum_{(i, j) \in E}\left(\overrightarrow{u_{j}}(t)-\overrightarrow{u_{i}}(t)\right), i=1,2, \ldots, n,
$$

which, for the kind of graphs we analyze in this work can be written as

$$
\overrightarrow{u_{i}}(t)=-\sum_{j=1}^{n} a_{i j}\left(\overrightarrow{u_{i}}(t)-\overrightarrow{u_{j}}(t)\right), i=1,2, \ldots, n
$$

This equation indicates that the evolution of the state of the node $i$ in time 
depends on the 'agreement' that this node reaches with all its nearest neighbors. It is obvious now that we can write 1 by using the Laplacian matrix of the graph:

$$
\begin{aligned}
& \overrightarrow{\dot{u}}(t)=-\mathcal{L} \vec{u}(t), \\
& \vec{u}(0)=\vec{z} .
\end{aligned}
$$

In the equation (3) the Laplacian matrix is acting over the vector $\vec{u}(t)$ which is updated in time. That is, $\vec{u}_{p}(t)$ is a scalar which represents the 'opinion' of the node $p$ at the time $t$. The solution of this equation is:

$$
\vec{u}(t)=e^{-t \mathcal{L}} \vec{z} .
$$

where $0=\mu_{1}<\mu_{2} \leq \cdots \leq \mu_{n}$ are the eigenvalues and $\vec{\psi}_{j, p}$ the $p$ th entry of the corresponding $j$ th eigenvector of the Laplacian matrix. Then, the solution of the consensus equation on the graph is given by

$$
u \overrightarrow{(t)}=e^{-t \mu_{1}}\left(\vec{\psi}_{1} \cdot \vec{z}\right) \vec{\psi}_{1}+e^{-t \mu_{2}}\left(\vec{\psi}_{2} \cdot \vec{z}\right) \vec{\psi}_{2}+\cdots+e^{-t \mu_{n}}\left(\vec{\psi}_{n} \cdot \vec{z}\right) \vec{\psi}_{n}
$$

where $\vec{x} \cdot \vec{y}$ represents the inner product of the corresponding vectors. When the time tends to infinity every node tends to the state dictated by the average of the values of the initial condition. This state is usually known as the consensus set [21] and it can be formally defined as the set $\mathcal{A} \subseteq \mathbb{R}^{n}$ which is the subspace $\operatorname{span}\{1\}$, i.e.,

$$
\mathcal{A}=\left\{\vec{u} \in \mathbb{R}^{n} \mid \overrightarrow{u_{i}}=\overrightarrow{u_{j}}, \forall i, j \in V\right\} .
$$

The following is a well-known result in the theory of consensus dynamics on networks.

Lemma 1. ([21] p. 46) Let $G$ be a connected graph. Then, the consensus dynamics converges to the agreement set with a rate of convergence that is dictated by $\mu_{2}$.

Proof. As $t \rightarrow \infty$

$$
\vec{u}(t) \rightarrow\left(\vec{\psi}_{1} \cdot \vec{z}\right) \vec{\psi}_{1}=\frac{\overrightarrow{1} \cdot \vec{z}}{n} \overrightarrow{1}
$$

and hence $\vec{u}_{t} \rightarrow \mathcal{A}$. As $\mu_{2}$ is the smallest positive eigenvalue of the graph Laplacian, it dictates the slowest mode of convergence in the equation 6.

For the sake of simulations it is sometimes useful to consider the discretetime model of consensus, whose equation can be written as follows [21, 20]: 


$$
\vec{u}_{i}(k+1)=\vec{u}_{i}(k)+\epsilon \sum_{j=1}^{n} a_{i j}\left(\vec{u}_{j}(k)-\vec{u}_{i}(k)\right),
$$

where $0<\epsilon<k_{\max }^{-1}$ is the time step for the simulation. The equation 9 can be written in matrix form as follows:

$$
\vec{u}(k+1)=(I-\epsilon L) \vec{u}(k),
$$

where $I$ is the identity matrix. The matrix $(I-\epsilon L)$ is usually known as the Perron matrix [20].

\section{Algebraic connectivity and diameter of RRGs}

As we have seen in the previous section the so-called algebraic connectivity $\mu_{2}[28,29]$ dictates the slowest mode of convergence in the consensus dynamics. That is, the rate at which a given group of nodes connected in a network reaches the global consensus is mainly determined by the second smallest eigenvalue of the Laplacian matrix. Consequently, we obtain the first result here, which is an upper bound for the algebraic connectivity of a RRG.

Theorem 2. Let $G_{R}(n, a, r)$ be a connected $R R G$ with $n$ nodes embedded in a rectangle of sides with lengths $a$ and $b=a^{-1}$, and connection radius $r$. Then, the algebraic connectivity, i.e., the second smallest eigenvalue of the Laplacian matrix, is bounded as

$$
\mu_{2}\left(G_{R}\right) \leq \frac{8(n-1)(a r)^{2}}{a^{4}+1} \log _{2}^{2} n .
$$

In order to prove Theorem 2 we need the following result.

Lemma 3. Let $G_{R}(n, a, r)$ be a connected $R R G$ with $n$ nodes embedded in a rectangle of sides with lengths a and $b=a^{-1}$, and two nodes are connected if and only if their at a Euclidean distance smaller or equal than $r$. Let $D=D\left(G_{R}\right)$ be the diameter of the corresponding $R R G$. Then,

$$
D\left(G_{R}\right) \geq \frac{\sqrt{a^{4}+1}}{a r} .
$$

Proof. The nodes of the RRG are uniformly and independently distributed in the unit rectangle. Then, let us assume that the $n$ points are equally spaced in the area of the rectangle separated by a Euclidean distance of $r$. In this case the largest number of points are connected along the main diagonal of the rectangle. If the length of the main diagonal is $c$ there are $\frac{c}{r}$ connected nodes in this line. Thus, the maximum shortest path distance in the RRG is $\frac{c}{r}$ with $c=\frac{\sqrt{a^{4}+1}}{a}$. For a connected RRG this is the shortest the diameter can be, because if two 
points in the main diagonal are separated at a Euclidean distance larger than $r$, then the diameter of $G_{R}$ will be larger than $\frac{c}{r}$, which proves the result.

Now, we consider the following bound obtained by Alon and Milman [30] for the algebraic connectivity of any simple graph.

Theorem 4. ([30]). The second smallest eigenvalue of the Laplacian matrix of any graph is bounded as

$$
\mu_{2}(G) \leq \frac{8 k_{\max }}{D^{2}} \log _{2}^{2} n .
$$

Then, by substituting 12 into 13 we have

$$
\mu_{2}(G) \leq \frac{8 k_{\max }}{d D^{2}} \log _{2}^{2} n \leq \frac{8 k_{\max }(a r)^{2}}{a^{4}+1} \log _{2}^{2} n \leq \frac{8(n-1)(a r)^{2}}{a^{4}+1} \log _{2}^{2} n,
$$

where the last inequality uses the fact that for any simple graph $k_{\max } \leq n-1$, which finally proves the Theorem 2 .

Remark 5. The results in this section prove that the elongation of the rectangle in the RRG makes the graphs drastically less connected for a given radius. However, as can be seen in the inequalities (11) and (12) the reduction of the algebraic connectivity with the rectangle elongation can be compensated with the increase of the connection radius, which also decreases the diameter of the graph. For instance, if we are considering the deployment of wireless sensors in very elongated region it is customary to use sensors which have coverage radius significantly larger than those typically used for covering more squared regions. Otherwise, there is a high risk that the whole network is disconnected.

\section{Consensus time}

Here we are interested in analyzing the influence of the algebraic connectivity on the time of consensus $t_{c}$, i.e., the time for which $\left|\overrightarrow{u_{i}}-\overrightarrow{u_{j}}\right| \leq \delta$, where $\delta$ is a given threshold. Then, we state our main result of this work.

Theorem 6. Let $G_{R}(n, a, r)$ be a connected $R R G$ with $n$ nodes embedded in a rectangle of sides with lengths $a$ and $b=a^{-1}$, and two nodes are connected if and only if they are at a Euclidean distance smaller than or equal to $r$. Let $\left\langle t_{c}\right\rangle$ be the time of consensus averaged for all the nodes in the graph. Let $\mu_{2}$ be the algebraic connectivity of the $R R G$ and $\vec{\psi}_{2}$ the corresponding Fiedler vector. Then

$$
\left\langle t_{c}\right\rangle \geq \frac{1}{n \mu_{2}} \sum_{p=1}^{n} \ln \left|\frac{\vec{\psi}_{2, p}\left(\vec{\psi}_{2} \cdot \vec{z}\right)}{\delta}\right|
$$


Proof. First, we write the eq. 6 for a given node $p$ as

$$
\vec{u}_{p}(t)=\sum_{q=1}^{n} \vec{z}_{q} \sum_{j=1}^{n} \vec{\psi}_{j, p} \vec{\psi}_{j, q} e^{-t \mu_{j}},
$$

which represents the evolution of the state of the corresponding node as time evolves. Now, let us consider that the time tends to the time of consensus $t \rightarrow t_{c}$, where $t_{c}$ is the time at which $u(t) \rightarrow\left(\vec{\psi}_{1}^{T} \vec{z}\right) \vec{\psi}_{1}$. Let us designate this time by $t_{c}^{-}$

$$
\vec{u}_{p}\left(t_{c}^{-}\right)=\frac{1}{n} \sum_{q=1}^{n} \vec{z}_{q}+\sum_{j=2}^{n}\left(\vec{\psi}_{j, p} e^{-t_{c}^{-}(p) \mu_{j}} \sum_{q=1}^{n} \vec{\psi}_{j, q} \vec{z}_{q}\right)
$$

here $t_{c}^{-}(p)$ means the time at which the node $p$ is close to reaching the consensus state. Let $\left\langle\vec{u}_{0}\right\rangle=\frac{1}{n} \sum_{q=1}^{n} \vec{z}_{q}$ and let us write 16 as follows

$$
\vec{u}_{p}\left(t_{c}^{-}\right)-\langle\vec{z}\rangle=\sum_{j=2}^{n}\left(\vec{\psi}_{j, p} e^{-t_{c}^{-}(p) \mu_{j}} \sum_{q=1}^{n} \vec{\psi}_{j, q} \vec{z}_{q}\right) .
$$

Let us select a node $p$ such that $\vec{\psi}_{2, p}$ has the same sign as $\vec{\psi}_{2} \cdot \vec{z}$.

Since $\mu_{2}$ corresponding to $j=2$ is the smallest eigenvalue in the sum on the right hand of the expression, this terms tends to 0 slower than the terms for the other values of $j$. This means that, if we choose a small enough value of $\delta$, the values of $t_{c}$ and thus $t_{c}^{-}$will be very large. Thus, we can ensure that the left side of the equation is small enough that $\sum_{j=3}^{n}\left(\overrightarrow{\psi_{j, p}} e^{-t_{c}^{-}(p) \mu_{j}}\left(\overrightarrow{\psi_{j}} \cdot \vec{z}\right)\right)<0$. This implies that

$$
\left(\vec{u}_{p}\left(t_{c}^{-}\right)-\langle\vec{z}\rangle\right)<\vec{\psi}_{2, p} e^{-t_{c}^{-}(p) \mu_{2}}\left(\vec{\psi}_{2} \cdot \vec{z}\right) .
$$

Now, because $\left|\vec{u}_{p}\left(t_{c}^{-}\right)-\langle\vec{z}\rangle\right| \geq \delta$ we have

$$
\delta \leq\left|\vec{u}_{p}\left(t_{c}^{-}\right)-\langle\vec{z}\rangle\right|<\left|\vec{\psi}_{2, p} e^{-t_{c}^{-}(p) \mu_{2}}\left(\vec{\psi}_{2} \cdot \vec{z}\right)\right| .
$$

Then, the time at which the consensus is reached $t_{c}(p)$ is bounded by

$$
t_{c}(p) \geq t_{c}^{-}(p) \geq \frac{1}{\mu_{2}} \quad \ln \left|\frac{\vec{\psi}_{2, p}\left(\vec{\psi}_{2} \cdot \vec{z}\right)}{\delta}\right| .
$$

Finally, the average time of consensus is bounded by 


$$
\left\langle t_{c}\right\rangle \geq \frac{1}{\mu_{2} n} \sum_{p=1}^{n} \ln \left|\frac{\vec{\psi}_{2, p}\left(\vec{\psi}_{2} \cdot \vec{z}\right)}{\delta}\right|,
$$

which proves the result.

If we are using a discrete-time approach like the one given in 10 then

$$
\left\langle t_{c}\right\rangle \geq \frac{1}{\epsilon \mu_{2} n} \sum_{p=1}^{n} \ln \left|\frac{\vec{\psi}_{2, p}\left(\vec{\psi}_{2} \cdot \vec{z}\right)}{\delta}\right| .
$$

The importance of the Theorem 6 is that when $\mu_{2} \rightarrow 0$ the time of consensus grows to infinity. Previously, we have already proved in Theorem 2 that the elongation of a random geometric graph with a given number of nodes and a fixed connection radius means that the algebraic connectivity goes asymptotically to zero. The immediate consequence of this result is that the consensus time grows to infinity in RRG when $a \rightarrow \infty$ due to the inverse relation between the consensus time and the algebraic connectivity.

\section{Simulations}

In this section we carry out simulations with the main goal of investigating how the elongation of a rectangle influences the consensus time. However, due to its importance for the current paper as well as in general for the further study of RRG we first investigate the influence of the rectangle side length on the diameter of the graphs and on their algebraic connectivity. Here we will consider RRGs constructed by placing $n=500$ nodes in a unit rectangle of side lengths $a$ and $b=a^{-1}$. The connection radius will be fixed to $r=0.15$ and we systematically vary the side length from $a=1$ to $a=12$. For each value of $a$ we take 100 random realizations of the RRG and report the average value of the corresponding property. In Fig. 2(a) we illustrate the plot of the average values of the diameter $\langle D\rangle$ versus the values of $a$ (blue squares). As can be seen the diameter increases linearly with the elongation of the rectangle. In fact, $\langle D\rangle \approx 7.927 a-0.237$. This result agrees with our analytical ones (see Theorem 3 ) which indicates that as $a \rightarrow \infty$ the diameter of the RRG also grows to infinity. The lower bound (12) is also plotted in Fig. 2(a) (red circles) where it can be seen that it follows identical trend as the observed value of the diameter for RRG. Indeed, the observed diameter linearly correlates with the one obtained by eq. 12 with a correlation coefficient of 0.999 .

The diameter is mainly used here to find an upper bound for the algebraic connectivity $\mu_{2}$ of the graph, i.e., the second smallest eigenvalue of the Laplacian matrix. We have calculated the average values of the algebraic connectivity for the RRGs studied here and the results are plotted in Fig. 2(b) (blue squares). 
As can be seen the algebraic connectivity decays as a power-law with the side length of the rectangle: $\mu_{2} \approx 0.7487 a^{-1.968}-0.00661$ with Pearson correlation coefficient equal to 0.9999. This confirms our analytical result that as $a \rightarrow \infty$ the algebraic connectivity decays to zero. We also include in this figure the plot corresponding to the values of the upper bound found for the algebraic connectivity (red circles). As can be seen, although the observed values are significantly smaller than the ones provided by the upper bound, they both decay following similar power-laws with the change of the rectangle side length. The observed values of the algebraic connectivity do not change linearly with those provided by the upper bound. Instead, they are related by a power-law relation of the type: $\left\langle\mu_{2}\right\rangle \approx 4476 \hat{\mu}_{2}^{0.61}-84.65$, where $\hat{\mu}_{2}$ is the upper bound obtained by the eq. (11).

The main conclusion of this part of the work is that when the rectangle becomes more elongated, the RRG becomes a larger world (the diameter increases) and also it displays less connectivity (decrease of $\mu_{2}$ ), which makes the graph more vulnerable to be split into isolated components by removing just a few nodes/edges. The consequences of this result for the analysis of the consensus dynamics are analyzed below.
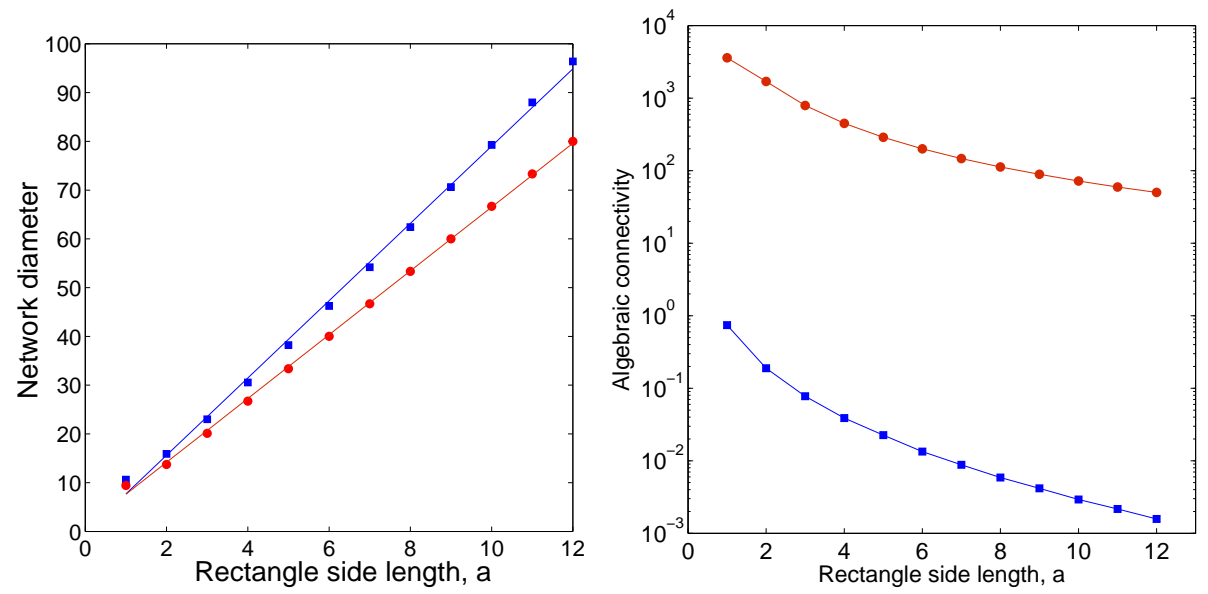

Figure 2: Change of the diameter (a) and the algebraic connectivity (b) of RRGs with the variation in the side length of the rectangle, $a$. All the graphs have $n=500$ nodes and the connection radius is $r=0.15$. The squares correspond to the average values observed for the RRG after 100 random realizations, and the circles represents the bounds obtained by eq. 12 and eq. 11, respectively. Notice the semilog plot on the y-axis for the plot (b).

We now study the influence of the rectangle elongation over the consensus dynamics on RRGs. We take care with the elongation process so that the graphs do not become disconnected. First we compare the discrete-time evolution of two RRGs with $n=500$ nodes and $r=0.15$, but one having $a=1$ (a 'classical' RGG) and the other having $a=5$. In Fig. 5 (a) and (b) we plot the time evolution of this consensus dynamics, where it can be seen that the time of 
consensus for the graph embedded into the unit square is at least 10 times shorter than that for the elongated RRG (see further). Because these plots are the results of only one random realization we perform a systematic variation of the rectangle side length and report the average of the time of consensus after 100 random realizations for each value of $a$ with a stopping criterion of $\delta=10^{-4}$. The results are illustrated in Fig. 5 (c), where we plot the values of the average time for consensus versus the rectangle side lengths (blue squares). As can be seen the time for consensus increases with the elongation of the rectangle. The best fit for this correlation is a 4 th order polynomial: $\left\langle t_{c}\right\rangle \approx$ $0.1885 a^{4}-1.651 a^{3}+19.59 a^{2}-37.06 a^{2}+30.59$; the fit has a Pearson correlation coefficient of 0.9997 . Using this model we can obtain a more precise estimation of the average time for consensus of the random realization illustrated in Fig. 5 (a) and (b). For a value of $\delta=10^{-4}$ the consensus is reached for $a=1$ at a time of 11.66 , while for $a=10$ at a time of 1853 . We will go back to this kind of analysis later on in this paper.

The estimated times for consensus obtained from the equation (21) are also plotted in Fig. 5 (c) (red circles), where it can be seen that they follow the same trend as the observed values. Indeed, the plot of the observed values versus those expected from the eq. (21) (see Fig. 5 (d)) indicates a perfect linear correlation between the two with a Pearson correlation coefficient of 0.9999 . 

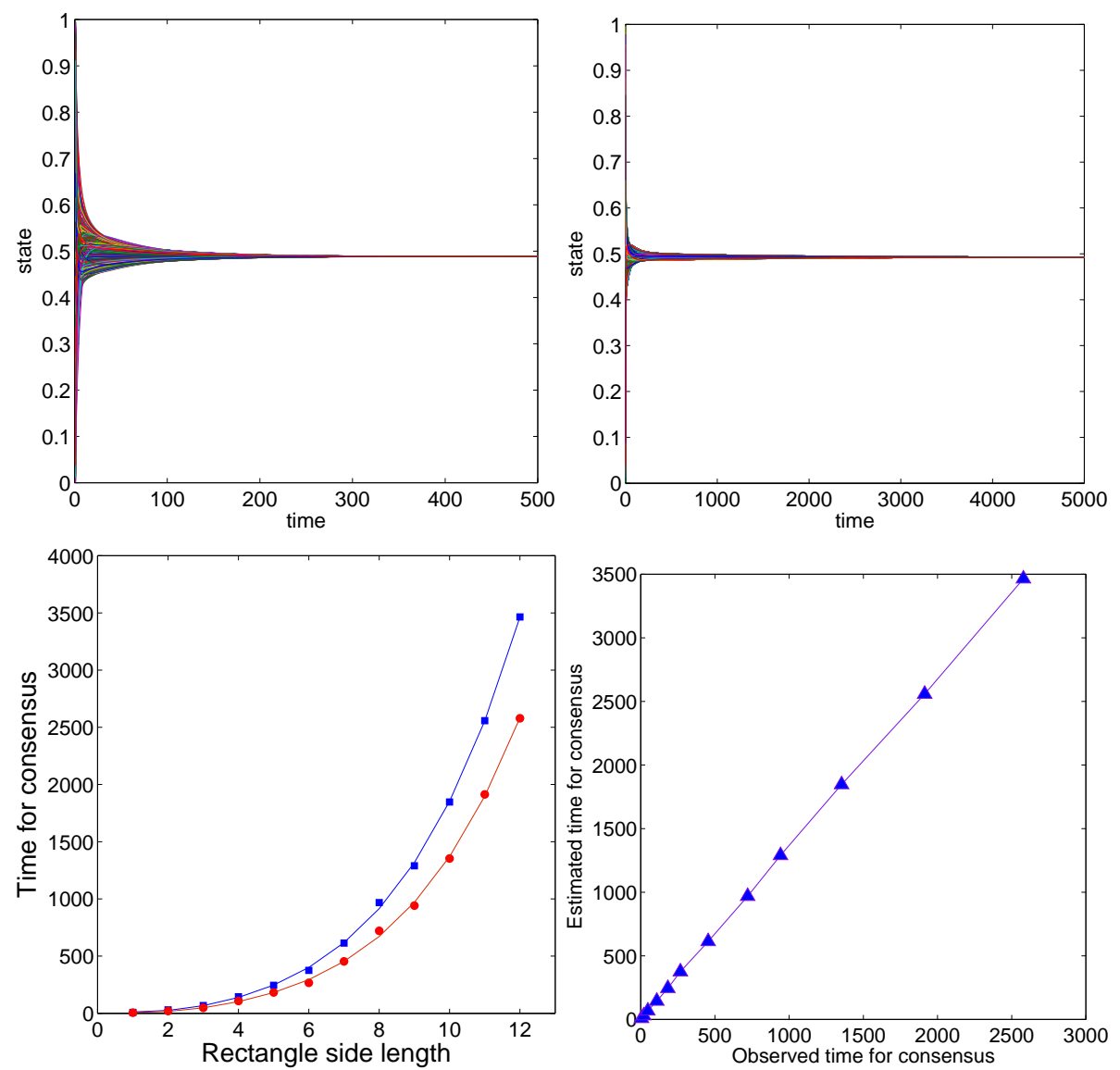

Figure 3: Illustration of the consensus dynamics for a RRG with $a=1$ (a) and for $a=5$ (b). The simulations were carried out using a discrete time consensus model (see 10) with a random allocation of initial states for the nodes. Both networks have 500 nodes and the connection radius is $r=0.15$. Notice that the scale for the time axis has changed by a factor of 10 from one plot to the other. (c) Dependence of the time for consensus with the length of the side of the rectangle. Here the squares represent the average values of the 100 simulations and the circles are the values obtained by the equation 21 . The solid lines represent the best fit which were obtained using 4th order polynomials. (d) Linear plot of the observed and estimated (using equation 21) for the time of consensus of the RRGs with 500 nodes and $r=0.15$.

Finally, we plot in Fig. 4 the dependence of the time of consensus with respect to both the connection radius and the rectangle side length. The line that divides the region of relatively fast consensus (deep blue region in the contour plot) from that of relatively slow one is given by $a=\kappa \cdot r-1.5$, where $\kappa=28$ for the analytical and $\kappa=26$ for the observed results. Thus, a condition for fast consensus in RRG with $n=500$ can be simply approximated by

$$
\frac{a+1.5}{r}<\kappa
$$


We would like to briey explore some of the consequences that our results have on the study of consensus in real-world situations. As we have seen in the Introduction a city like Manhattan has dimensions which resemble a rectangle more than a square. That is, Manhattan is $21.6 \mathrm{~km}$ long and $3.7 \mathrm{~km}$ wide. This can be represented as a unit rectangle of dimensions $a \approx 2.42$ and $b \approx 0.41$. Using our fitted model, and considering that we embed 500 nodes, e.g., wireless sensors to monitor the city, we obtain the expected time for consensus on this $\mathrm{RRG}$, which is 38.3. This time is 3.3 times longer than the one expected if the network is considered to be embedded into a unit square, i.e., $a=1$. That is, we would be underestimating the time for consensus of the sensors by a factor of three. Also, according to (23) we can estimate that a fast consensus can be reached in this network only if $r>0.157$.
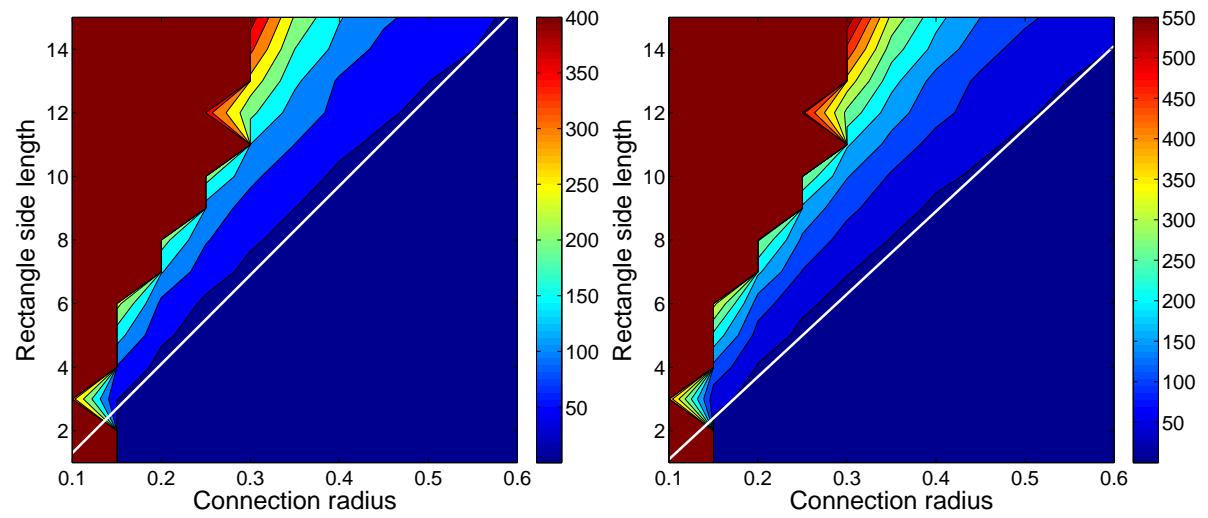

Figure 4: Contour plot showing the dependence of the time of consensus with the connection radius and the rectangle side length in RRGs with 500 nodes. a) Analytical results. b) Observed results from the simulations. The diagonal white line corresponds to the equations $a=\kappa \cdot r-1.5$, where $\kappa=28$ for the analytical and $\kappa=26$ for the observed results.

\section{Conclusions}

In this work we have found some interesting structural and dynamical properties of graphs embedded into rectangular areas. The recently defined random rectangular graphs (RRGs) account for the spatial distribution of nodes allowing the variation of the shape of the unit rectangle commonly used in random geometric graphs (RGGs). In particular, we have found an excellent lower bound for the diameter of RRGs. The diameter is an important parameter per se as well as for its inclusion in many inequalities for other network structural parameters. For instance, we have used this bound to find an upper bound for the algebraic connectivity of RRGs. The algebraic connectivity, the second smallest eigenvalue of the graph Laplacian, is one of the most important parameters relating network structure and dynamical processes taking place on them, e.g., consensus/diffusion dynamics, synchronization. Finally, we have studied the consensus dynamics on RRGs where we have found analytically that as the 
rectangle becomes more elongated, the time for reaching consensus increases polynomially with the side length of the rectangle. The simulation results allowed us to confirm these results and to find empirical relations between the topological and dynamical parameters with the rectangle side length.

The results obtained in this work have important practical consequences for modeling real-world scenarios. First, modeling a real-world scenario which is not geometrically similar to a square using the 'classical' RGG, produces a significant error in estimating important structural and dynamical network parameters. More importantly, the RRG provides a modeling scenario in which we can simulate the influence of the shape of a geographical region on the topological and dynamical properties of the graphs embedded on them.

There are many new research avenues that the study of RRGs open for the study of spatially embedded graphs. One of them is the analysis of other dynamical processes, such as synchronization, and epidemic spreading on RRGs. Another area of development is the extension of RRGs to higher dimensions, specially to three-dimensional (3D) ones. 3D-RRGs will allow the effective modeling of many real-world scenarios in which the nodes are embedded into elongated cubic regions of the $3 \mathrm{D}$ space. Finally, a third area of interesting development is the consideration of other proximity graphs, such as Gabriel graphs and random neighborhood graphs, embedded into rectangular regions instead of unit squared ones. We hope these developments will contribute to be better understanding of networks embedded into geometrical spaces.

[1] Marc Barthélemy. Spatial networks. Physics Reports, 499(1):1-101, 2011. doi: doi:10.1016/j.physrep.2010.11.002.

[2] I.F. Akyildiz and M.C. Vuran. Wireless Sensor Networks. Advanced Texts in Communications and Networking. Wiley, 2010. ISBN 9780470515198. URL http://books.google.co.uk/books?id=7YBHYJsSmS8C.

[3] E. Estrada. The Structure of Complex Networks: Theory and Applications. OUP Oxford, 2011. ISBN 9780191613425. URL http: //books.google.co.uk/books?id=6iK4vp8oewYC.

[4] Elizabeth Santiago, Jorge X. Velasco-Hernández, and Manuel RomeroSalcedo. A methodology for the characterization of flow conductivity through the identification of communities in samples of fractured rocks. Expert Systems with Applications, 41(3):811 - 820, 2014. ISSN 0957-4174. doi: http://dx.doi.org/10.1016/j.eswa.2013.08.011. URL http://www. sciencedirect. com/science/article/pii/S0957417413006234. Methods and Applications of Artificial and Computational Intelligence.

[5] Andrea Perna, Sergi Valverde, Jacques Gautrais, Christian Jost, Ricard Solé, Pascale Kuntz, and Guy Theraulaz. Topological efficiency in three-dimensional gallery networks of termite nests. Physica A: Statistical Mechanics and its Applications, 387(24):6235 - 6244, 2008. ISSN 0378-4371. doi: http://dx.doi.org/10.1016/j.physa.2008.07.019. URL http://www.sciencedirect.com/science/article/pii/S0378437108006675. 
[6] J. Buhl, J. Gautrais, R.V. Solé, P. Kuntz, S. Valverde, J.L. Deneubourg, and G. Theraulaz. Efficiency and robustness in ant networks of galleries. The European Physical Journal B - Condensed Matter and Complex Systems, 42(1):123-129, 2004. ISSN 1434-6028. doi: 10.1140/epjb/e200400364-9. URL http://dx.doi.org/10.1140/epjb/e2004-00364-9.

[7] Dean Urban and Timothy Keitt. Landscape connectivity: a graph-theoretic perspective. Ecology, 82(5):1205-1218, 2001. doi: 10.1890/0012-9658.

[8] Mathew Penrose. Random geometric graphs, volume 5. Oxford University Press Oxford, 2003. ISBN 01985062609780198506263.

[9] Jesper Dall and Michael Christensen. Random geometric graphs. Phys. Rev. E, 66:016121, Jul 2002. doi: 10.1103/PhysRevE.66.016121. URL http://link.aps.org/doi/10.1103/PhysRevE.66.016121.

[10] B. Bollobás. Random Graphs. Academic Press, London, 1985. URL http://www. amazon. com/Random-Graphs-Bela-Bollobas/dp/0121117561.

[11] E. N. Gilbert. Random graphs. Ann. Math. Statist., 30 (4):1141-1144, 12 1959. doi: 10.1214/aoms/1177706098. URL http://dx.doi.org/10.1214/aoms/1177706098.

[12] Piyush Gupta and P.R. Kumar. Critical power for asymptotic connectivity in wireless networks. In WilliamM. McEneaney, G.George Yin, and Qing Zhang, editors, Stochastic Analysis, Control, Optimization and Applications, Systems \& Control: Foundations \& Applications, pages 547-566. Birkhüuser Boston, 1999. ISBN 978-1-4612-7281-6. doi: 10.1007/978-1-4612-1784-8_33. URL http://dx.doi.org/10.1007/978-1-4612-1784-8_33.

[13] Deborah Estrin, Ramesh Govindan, John Heidemann, and Satish Kumar. Next century challenges: Scalable coordination in sensor networks. In Proceedings of the 5th Annual ACM/IEEE International Conference on Mobile Computing and Networking, MobiCom '99, pages 263-270, New York, NY, USA, 1999. ACM. ISBN 1-58113-142-9. doi: 10.1145/313451.313556. URL http://doi.acm.org/10.1145/313451.313556.

[14] G. J. Pottie and W. J. Kaiser. Wireless integrated network sensors. Commun. ACM, 43(5):51-58, May 2000. ISSN 0001-0782. doi: 10.1145/332833.332838. URL http://doi.acm.org/10.1145/332833.332838.

[15] H. Kenniche and V. Ravelomananana. Random geometric graphs as model of wireless sensor networks. In Computer and Automation Engineering (ICCAE), 2010 The 2nd International Conference on, volume 4, pages 103-107, Feb 2010. doi: 10.1109/ICCAE.2010.5451758. 
[16] M. Franceschelli, A. Giua, and C. Seatzu. Distributed averaging in sensor networks based on broadcast gossip algorithms. Sensors Journal, IEEE, 11(3):808-817, March 2011. ISSN 1530-437X. doi: 10.1109/JSEN.2010.2064295.

[17] Albert Díaz-Guilera, Jesús Gómez-Gardeñes, Yamir Moreno, and Maziar Nekovee. Synchronization in random geometric graphs. International Journal of Bifurcation and Chaos, 19 (02):687-693, 2009. doi: 10.1142/S0218127409023044. URL http: //www . worldscientific.com/doi/abs/10 .1142/S0218127409023044.

[18] Andrea Baronchelli and Albert Díaz-Guilera. Consensus in networks of mobile communicating agents. Phys. Rev. E, 85: 016113, Jan 2012. doi: 10.1103/PhysRevE.85.016113. URL http://link.aps.org/doi/10.1103/PhysRevE.85.016113.

[19] S.S. Pereira and A. Pages-Zamora. Consensus in correlated random wireless sensor networks. Signal Processing, IEEE Transactions on, 59(12):62796284, Dec 2011. ISSN 1053-587X. doi: 10.1109/TSP.2011.2166552.

[20] R. Olfati-Saber, J.A. Fax, and R.M. Murray. Consensus and cooperation in networked multi-agent systems. Proceedings of the IEEE, 95(1):215-233, Jan 2007. ISSN 0018-9219. doi: 10.1109/JPROC.2006.887293.

[21] M. Mesbahi and M. Egerstedt. Graph Theoretic Methods in Multiagent Networks. Princeton Series in Applied Mathematics. Princeton University Press, 2010. ISBN 9781400835355. URL http: //books.google.co.uk/books?id=GlrqRwumdDcC.

[22] Valerie Isham, Joanna Kaczmarska, and Maziar Nekovee. Spread of information and infection on finite random networks. Phys. Rev. E, 83:046128, Apr 2011. doi: 10.1103/PhysRevE.83.046128. URL http://link.aps.org/doi/10.1103/PhysRevE.83.046128.

[23] Zoltán Toroczkai and Hasan Guclu. Proximity networks and epidemics. Physica A: Statistical Mechanics and its Applications, 378(1):68 - 75, 2007. ISSN 0378-4371. doi: http://dx.doi.org/10.1016/j.physa.2006.11.088. URL http://www.sciencedirect.com/science/article/pii/S0378437106012672. Social network analysis: Measuring tools, structures and dynamics Social Network Analysis and Complexity.

[24] Maziar Nekovee. Worm epidemics in wireless ad hoc networks. New Journal of Physics, 9(6):189, 2007. doi: 10.1088/1367-2630/9/6/189. URL http: //stacks. iop.org/1367-2630/9/i=6/a=189.

[25] Steven Riley, Ken Eames, Valerie Isham, Denis Mollison, and Pieter Trapman. Five challenges for spatial epidemic models. Epidemics, (0), 2014. ISSN 1755-4365. doi: http://dx.doi.org/10.1016/j.epidem.2014.07.001. URL http://www.sciencedirect.com/science/article/pii/S1755436514000310. 
[26] Weituo Zhang, Chjan C Lim, Gyorgy Korniss, and Boleslaw K Szymanski. Opinion dynamics and influencing on random geometric graphs. Scientific reports, 4, 2014. doi: 10.1038/srep05568.

[27] Ernesto Estrada and Matthew Sheerin. Random rectangular graphs. arXiv preprint arXiv:1502.02577, 2015.

[28] Miroslav Fiedler. Algebraic connectivity of graphs. Czechoslovak Mathematical Journal, 23(2):298-305, 1973.

[29] Nair Maria Maia de Abreu. Old and new results on algebraic connectivity of graphs. Linear Algebra and its Applications, 423(1):53 - 73, 2007. ISSN 0024-3795. doi: http://dx.doi.org/10.1016/j.laa.2006.08.017. URL http://www.sciencedirect.com/science/article/pii/S0024379506003971. Special Issue devoted to papers presented at the Aveiro Workshop on Graph Spectra Aveiro Workshop on Graph Spectra.

[30] N Alon and V.D Milman. $\lambda 1$, isoperimetric inequalities for graphs, and superconcentrators. Journal of Combinatorial Theory, Series B, 38(1):73 - 88, 1985. ISSN 0095-8956. doi: http://dx.doi.org/10.1016/0095-8956(85)90092-9. URL http://www. sciencedirect. com/science/article/pii/0095895685900929. 